\title{
Acciones ambientales: Responsabilidad Social en MiPyMes
}

\section{Environmental actions: Social Responsibility in MSMEs}

\author{
ACEVES-LÓPEZ, Jesús Nereida, GONZÁLEZ-NAVARRO, Nora Edith y NAVARRO-ARVIZU, \\ Elba Myriam
}

Instituto Tecnológico de Sonora, 5 de Febrero \#818 sur, Colonia Centro, Ciudad Obregón, Sonora.

ID $1^{\mathrm{er}}$ Autor: Jesús Nereida, Aceves López / ORC ID: 0000-0003-2442-0686

ID $1^{\mathrm{er}}$ Coautor: Nora Edith, González Navarro / ORC ID: 0000-0002-7112-8082

ID $2^{\text {do }}$ Coautor: Elba Myriam, Navarro Arvizu / ORC ID: 0000-0003-2695-0883

DOI: $10.35429 / J U S D .2019 .15 .5 .24 .30$

Recibido: 30 de Marzo, 2019; Aceptado 30 de Junio, 2019

Resumen

La atención al medio ambiente es de urgencia mundial y es compromiso de todos los sectores. El objetivo de esta investigación es identificar el desempeño del sector empresarial respecto al cuidado del medio ambiente. Planteándose la siguiente pregunta de investigación, ¿Cuáles son las acciones sociales que realizan las empresas respecto a su medio ambiente? Se llevó a cabo un estudio no experimental, correspondiente a 88 MiPyMes de Cd. Obregón, Sonora. Aplicándose cuestionario conformados por 18 preguntas, algunas de ellas con opción múltiple, medidas en una escala de Likert del 1 al 5, dividido en tres Secciones: I. Datos del responsable del negocio, II. Generalidades de la empresa y III. Acciones ambientales en la empresa. Los resultados obtenidos muestran que aunque ya se realizan ciertas actividades, aún existe mucho trabajo por hacer, en acciones como: utilización de empaquetados ecológicos y/o reciclables; adopción de medidas en cuanto al diseño de productos y/o servicios ecológicos; consideración de programas para uso de energías alternativas, y acceder voluntariamente a las regulaciones ambientales. Los resultados obtenidos permitirán diseñar programas, donde los empresarios adquieran conocimientos, que les ayuden a desarrollar estrategias ambientales a través de acciones de responsabilidad social, generando un valor agregado a la misma organización.

Sustentabilidad, Responsabilidad Social, Medio ambiente

\begin{abstract}
The attention to the environment is of global urgency and is a commitment of all sectors. The objective of this research is to identify the performance of the business sector regarding the care of the environment. Posing the following research question, what are the social actions carried out by companies with respect to their environment? A non-experimental study was carried out, corresponding to $88 \mathrm{MiPyMes}$ of Cd. Obregón, Sonora. Applying questionnaire consisting of 18 questions, some of them with multiple choice, measured on a Likert scale from 1 to 5, divided into three Sections: I. Data of the person in charge of the business, II. General aspects of the company and, III. Environmental actions in the company. The results obtained show that although certain activities are already being carried out, there is still a lot of work to be done, in actions such as: use of ecological and / or recyclable packaging; adoption of measures regarding the design of ecological products and / or services; Consideration of programs for the use of alternative energies, and voluntary access to environmental regulations. The results obtained will allow designing programs, where entrepreneurs acquire knowledge, which will help them develop environmental strategies through social responsibility actions, generating added value to the same organization.
\end{abstract}

Sustainability, Social responsibility, Environment

Citación: ACEVES-LÓPEZ, Jesús Nereida, GONZÁLEZ-NAVARRO, Nora Edith y NAVARRO-ARVIZU, Elba Myriam. Acciones ambientales: Responsabilidad Social en MiPyMes. Revista del Desarrollo Urbano y Sustentable. 2019. 5-15: 2430

\footnotetext{
* Correspondencia del Autor (nereida.aceves@itson.edu.mx)

$\dagger$ Investigador contribuyendo como primer autor
} 


\section{Introducción}

El tema ambiental es uno de los aspectos que tiene al mundo en gran preocupación, el alto grado de contaminación obliga a ponerle atención, es un tema de carácter mundial, donde cada uno de los individuos tiene su responsabilidad, cada acción personal tiene un gran impacto en el mundo actual. Es en este punto que los países están promoviendo acciones que generen resultados positivos en bosques, océanos, entre otros, pues de seguir en este ritmo acelerado de industrialización se pronostica traerá consecuencias devastadoras en el hábitat.

Las empresas son parte fundamental de ello, por lo que éstas deberán ser capaces de implementar acciones internas que mejoren las condiciones ambientales. En este trabajo de investigación se muestra la situación actual de empresas de Cd. Obregón, Sonora, respecto al conocimiento y acciones que llevan o no cabo respecto a la responsabilidad Social.

\section{Justificación}

Es urgente que todos los actores de los sectores económicos respondan a estos nuevos contextos, donde imperan los cambios climáticos, aspectos culturales, sociales y de género, cuyos efectos en cada uno de ellos, genera una acción y reacción en cadena: desde los integrantes de la familia hasta los componentes de los grandes industrias, y líderes mundiales, el compromiso debe ser tomado por todos.

De lo anterior surge un tema conocido como Responsabilidad Social aspecto en el cual cada vez más la entidad es un actor dinámico, pues si bien es cierto que la responsabilidad no es absoluta de la empresa, sin duda alguna su desempeño tiene un fuerte impacto en la sociedad. En esta temática las MiPyMes tienen varias debilidades, que es imperante se empiecen a abordar, de tal forma que sus dirigentes puedan llevar a cabo planes donde se incluyan estos nuevos aspectos, como es la sustentabilidad, responsabilidad social.

\section{Planteamiento de problema}

Las empresas además de tener la responsabilidad de ser productivas y generar riqueza, deben responder a las exigencias del entorno en que éstas se desarrollan. Y uno de los grandes problemas a los que el mundo se está enfrentando son los cambios climáticos, situación generada por los daños ambientales a los que ha contribuido el ser humano, los cuales se caracterizan por: ser continuos, acumulativos, irreversibles, transfronterizos (flora, fauna, ambiente, personas). (Sanchez, citado por Perez 2009).

El sector empresarial deberá de llevar a cabo procesos donde incluya acciones de responsabilidad social, no solo en su aspecto interno: como son empleados, accionistas, dueños, , sino que deberá de atender al aspecto externo: trabajar con sus proveedores, clientes, gobierno y sobre todo a la comunidad en la que se encuentra, esto implica al interior de las organizaciones grandes cambios, para los cuales se requiere conocimiento claro de las responsabilidades y derechos sobre ello, como es el caso de la responsabilidad social, si bien es cierto ,los empresarios manifiestan que ya hay una conciencia social, sobre todo en cuestión ambiental en el tema de reciclaje, donde se observa que han avanzado, sin embargo todavía existen muchos aspectos que requieren de forma urgente trabajarse, por ello es importante que los empresarios o dirigentes empresariales inicien con este proceso de aprendizaje sobre estas nuevas exigencias, y para eso primero es indispensable la identificación de su situación actual en estos términos.

\section{Objetivo}

Determinar las acciones de RS, que llevan a cabo las MiPyMes de Cd. Obregón, Sonora, respecto al medio ambiente, esto a través de un diagnóstico.

\section{Marco teórico}

Es a finales de 960 e inicios de 1970, que el tema de crisis ambiental se incluyó en los aspectos políticos de los países, esto debido al gran número de reportes científicos que informaron sobre el agotamiento de los recursos naturales, el riesgo ambiental propiciado por la misma humanidad. 
Lo anterior se incrementó debido a la extracción de recursos naturales, así como el incremento de desechos que se generan por los procesos productivos. (Pierri, 2005, Arroyo, Camarero \&Vasquez, 1997), citados por (Gómez, 2014).

"A partir de las preocupaciones precedentes, en 1972 se inició un periodo que tardaría cerca de veinte años durante el cual se consolidaría el concepto de desarrollo sostenible, cuyos esfuerzos se plasmarían, inicialmente, en la Declaración de Estocolmo (1972) y concluirían con la Declaración de Río de Janeiro sobre el Medio Ambiente y el Desarrollo en 1992, así como el Programa 21, bajo el cual se desarrolló y consolidó la noción contemporánea de desarrollo sostenible (Romero, 2012, p. 47), citado por (Salamanca 2016).

El término desarrollo sustentable, es cada vez más utilizado, y ello tiene que ver con los recursos naturales. El desarrollo sustentable o sostenible, es definido como aquel que satisface las necesidades de las generaciones presentes sin perjudicar la capacidad de las generaciones futuras en satisfacer sus propias necesidades, (Robles, Encinas 2014).

De lo anterior se señala que México, llegaría ser sustentable, cuando sea capaz de lograrlo cuando lleve a cabo el manejo sustentable de: de los recursos naturales, de la sociedad y de la economía.

Wolfensberger ( 2005), menciona que la naturaleza no puede ser observada como algo ajeno y aislado de las personas, situación que es agravada por la despiadada explotación de los recursos naturales, generando una destrucción a todo los ecosistemas, lo cual se ve afectado por el daño al aspecto social, ello por las situaciones de violencia que se están viviendo. Al respecto esta autora señala que se debe comprender a la naturaleza como energía unitaria por la cual se mueve todo, donde se debe ir con ella y no en contra de ella, donde al tener sabiduría ecológica y el conocimiento de ecotecnias se puede contribuir al cuidado del medio ambiente.
Lo anterior muestra hoy en día, la exigencia de una educación ambiental, donde se formen individuos dotados de conocimientos, habilidades, y actitudes, que permitan realizar acciones de cambio para la solución de los retos que el ser humano está enfrentando, es decir formar personas consientes y sensibles a su medio ambiente.

El mundo empresarial cada vez enfrenta más exigencias, ya que no solo es responsable de ser productivas y generar riqueza, sino que además éstas a través de sus productos y/o servicios deberán de mejorar la situación y bienestar de la sociedad en la que ésta se desempeña, es decir deberán ser responsables socialmente, lo que ha dado paso al interés por conceptos como Responsabilidad Social (RS). El consejo Europeo de Lisboa, se dirigió a las empresas haciendo hincapié en la responsabilidad en aspectos como: formación, organización del trabajo, nuevas oportunidades, inclusión social y desarrollo sostenido. Señalando que esta responsabilidad debe ser voluntaria, para lo cual se requiere de nuevas formas de trabajo que permitan reforzar la cohesión social, económica, salud y cuidado del medio ambiente, entre otras. ( López, 2002).

La Responsabilidad Social Empresarial (RSE), es definida por el libro verde como: la inclusión voluntaria por parte de las entidades, de las preocupaciones sociales y medioambientales en cada una de las actividades desarrolladas provenientes de sus operaciones. (libro verde 2001). Cajiga 2011, citado por (López, Gil, 2014), señala que la RSE, "Es el compromiso consciente y congruente de cumplir de manera integral con la finalidad de la empresa, en lo interno como lo externo, considerando las expectativas económicas, sociales y ambientales de todos sus participantes, demostrando respeto por las personas, los valores éticos, la comunidad y el medio ambiente".

Al respecto Raufflet (2011), señala que las empresas no son totalmente responsables de lo que en su entorno sucede, solo lo es de algunos aspectos, y la percepción y práctica de la RSE, y ésta puede cambiar con el transcurso del tiempo. 
Para la Unión Europea la RSE, se contiene de dos dimensiones: a) interna a la entidad, a la que le corresponden las áreas de recursos humanos, salud y seguridad en el trabajo, adaptación al cambio tecnológico y de la organización, respecto al medio ambiente ésta solo en lo que le afecta a la empresa en sus propios procesos. b) exterior a la empresa, ésta se refiere a las relaciones que lleva a cabo la organización respecto a la comunidad en la que se desempeña, así mismo con sus clientes y proveedores, señala también el tema de derechos humanos y medio ambiente pero en el aspecto de carácter mundial. (Robles, Encinas ,2014).

\section{Metodología}

\section{Tipo y diseño}

La investigación que a continuación se presenta es del tipo no experimental transaccional, lo anterior debido a que no se manipulan las variables bajo estudio, donde solo se describirá información de la situación que guarda la empresa en el tiempo del trabajo que se haga en ella, porque la situación de los participantes analizados no será alterada. Participantes

Esta investigación se realizó en 88 empresas de Cd. Obregón, Sonora, México, de los sectores de comercio, servicios e industrial. El estudio se refiere a identificar cuáles son las acciones de responsabilidad social que llevan a cabo las empresas con su medio ambiente.

\section{Materiales}

A través de la entrevista y con un cuestionario estructurado, fue el método que se utilizó en esta investigación. El instrumento de medición fue adaptado de Herrera, Larrán, Lechuga y Martínez (2016).

$$
\text { Se aplicó un }
$$

cuestionario dividido en tres secciones: I Datos del responsable del negocio, el cual contiene seis ítems; Sección II: Generalidades de la empresa, compuesta por tres ítems; y la sección III: denominado medioambiente está conformado por nueve ítems, la escala de medición de licker fue de 5 a 1 .

\section{Procedimiento}

1. Investigación documental en relación a los temas de sustentabilidad, responsabilidad social y el instrumento a aplicar en la investigación.

2. Revisión y adaptación del instrumento a aplicar.

3. Análisis de la población que se invitará a participar en proyecto.

4. Visita a los empresarios, para presentar el proyecto y solicitar su participación en el proyecto.

5. Determinación de muestra a partir de la aceptación del empresario.

6. Aplicación del instrumento a la muestra seleccionada.

7. Análisis y presentación de resultados

\section{Resultados}

\section{Sección I. Datos del entrevistado}

En este apartado se observó que: 67 son dueños, siendo el $76.1 \%$, cinco son directivos siendo el $5.7 \%, 14$ son gerentes siendo el $15.9 \%$ y dos no respondieron a la pregunta siendo $2.3 \%$. En relación al sexo, 35 personas respondieron son femenino siendo el $39.8 \%$ y 53 personas, que son de sexo masculino, siendo así el $60.2 \%$. En relación a la edad: 12 Personas están en el rango de 18 a 25 años, siendo el $13.6 \%$ de los encuestados, 32 personas están en el rango de 26 a 40 años, siendo el 36.4\%, 39 personas están en el rango de 41 a 60 años, siendo el $44.3 \%$ y dos personas están en el rango de 60 años en adelante, siendo el $2.3 \%$, tres no respondieron a la pregunta correspondiendo al $3.4 \%$.

En respuesta a la antigüedad de trabajo en la empresa: 14 personas están en el rango de 0 a 6 meses de antigüedad, representando $15.9 \%, 21$ personas están en el rango de 7 meses a 1 año de antigüedad, siendo el $23.9 \%$, 22 respondieron que tienen de 2 a 5 años de antigüedad, siendo el 25\%, 13 respondieron que tienen de 6 a 10 años siendo el $14.8 \%, 15$ personas respondieron que tienen de 11 años en delante de antiguiedad, siendo el $17 \%$ y tres personas no contestaron la pregunta siendo $3.4 \%$. 
Respecto a su estado civil. 28 personas respondieron que son solteros, siendo el $31.8 \%$ de los encuestados, 54 personas contestaron que son casados, siendo el $61.4 \%$, tres personas respondieron que son divorciados, siendo el $3.4 \%$, tres personas respondieron que están en unión libre siendo el $3.4 \%$.

En relación al nivel de estudios, una persona respondió que su estudios son hasta primaria, siendo el $1.1 \%$ de los encuestados, 15 personas respondieron que su nivel de estudios es secundaria siendo el $17 \%$ de los encuestados, 13 respondieron que su nivel es técnico siendo el $14.8 \%, 57$ personas respondieron que su nivel de estudios es profesional siendo el $64.8 \%$ $\mathrm{y}$ dos personas no respondieron, siendo el $2.3 \%$.

\section{Sección II. Generalidades de la empresa}

En relación a la estructura de la propiedad de la empresa: 51 personas respondieron que su empresa es de estructura familiar, siendo el $58 \%, 34$ respondieron que su empresa no es de estructura familiar, siendo el $38.6 \%$ y tres personas no respondieron la pregunta, siendo el 3.4\%. En relación al número de empleados, 74 respondieron que están en el rango de 0 a 10 , siendo el $84.1 \%$, ocho personas respondieron que están en el rango de 11 a 50 empleados, siendo el $9.1 \%$ y seis restantes no contestaron a la pregunta, siendo el $6.8 \%$. Respecto al sector 37 pertenecen al sector comercial, de las cuales de diez son del giro de abarrotes, siendo $27 \%$, ferretería tres, siendo el $8.1 \%$, en venta de ropa es de ocho, siendo un $21.6 \%$ y en otros giros se identifican 16 con un porcentaje del $43.2 \%$. El sector servicios se compone de 40 negocios, representando el $45 \%$ del total de los encuestados.

\section{Sección III. Medio Ambiente}

\begin{tabular}{|c|l|r|r|}
\cline { 3 - 4 } \multicolumn{1}{c|}{} & Frecuencia & Porcentaje \\
\hline \multirow{4}{*}{ Válidos } & Nunca & 14 & 15.9 \\
\cline { 2 - 4 } & casi nunca & 6 & 6.8 \\
\cline { 2 - 4 } & a veces & 15 & 17.0 \\
\cline { 2 - 4 } & Casi siempre & 11 & 12.5 \\
\cline { 2 - 4 } & Siempre & 41 & 46.6 \\
\cline { 2 - 4 } & No contestó & 1 & 1.1 \\
\cline { 2 - 4 } & Total & 88 & 100.0 \\
\hline
\end{tabular}

Tabla 2 Reutiliza y recicla materiales Fuente: Elaboración Propia
En relación al reciclaje, 14 personas respondieron que nunca reutilizan y reciclan materiales, siendo el $15.9 \%$, seis personas respondieron que casi nunca lo hacen, correspondiendo al $6.8 \%, 15$ personas respondieron que a veces lo hacen, correspondiendo al 17\%, 11 personas respondieron que casi siempre, correspondiendo al $12.5 \%, 41$ personas respondieron que siempre, correspondiendo al $46.6 \%$ y una persona no respondió siendo el $1.1 \%$.

\begin{tabular}{|c|l|r|r|}
\hline \multicolumn{5}{|c}{} & Frecuencia & Porcentaje \\
\hline \multirow{4}{*}{ Válidos } & Nunca & 2 & 2.3 \\
\cline { 2 - 4 } & casi nunca & 12 & 13.6 \\
\cline { 2 - 4 } & a veces & 16 & 18.2 \\
\cline { 2 - 4 } & Casi siempre & 22 & 25.0 \\
\cline { 2 - 4 } & Siempre & 36 & 40.9 \\
\cline { 2 - 4 } & Total & 88 & 100.0 \\
\hline
\end{tabular}

Tabla 3 Minimiza el impacto medioambiental de sus actividades

Fuente: Elaboración Propia

Dos personas respondieron que nunca minimizan el impacto, siendo el 2.3\%, 12 personas respondieron que casi nunca lo hacen, correspondiendo al $13.6 \%, 16$ respondieron que a veces, correspondiendo al 18.2\%, 22 personas respondieron que casi siempre, correspondiendo al $25 \%$ y 36 personas respondieron que siempre correspondiendo al $40.9 \%$.

\begin{tabular}{|c|l|r|r|}
\multicolumn{2}{c|}{} & Frecuencia & Porcentaje \\
\hline \multirow{5}{*}{ Válidos } & Nunca & 9 & 10.2 \\
\cline { 2 - 4 } & casi nunca & 10 & 11.4 \\
\cline { 2 - 4 } & a veces & 14 & 15.9 \\
\cline { 2 - 4 } & Casi siempre & 19 & 21.6 \\
\cline { 2 - 4 } & Siempre & 34 & 38.6 \\
\cline { 2 - 4 } & No contestó & 2 & 2.3 \\
\cline { 2 - 4 } & Total & 88 & 100.0 \\
\hline
\end{tabular}

Tabla 4 Realiza inversiones para ahorrar energía Fuente: Elaboración Propia

Inversiones para ahorrar energía, nueve personas respondieron que nunca las realizan, siendo el $10.2 \%$, diez personas respondieron que casi nunca lo hacen, correspondiendo al $11.4 \%, 14$ personas respondieron que a veces, correspondiendo al 15.9\%, 19 personas respondieron que casi siempre, correspondiendo al $21.6 \%, 34$ personas respondieron que siempre, correspondiendo al $38.6 \%$ y dos personas no respondieron a la pregunta siendo el $2.3 \%$. 


\begin{tabular}{|c|c|c|c|}
\hline & & Frecuencia & Porcentaje \\
\hline \multirow{6}{*}{ Válidos } & Nunca & 16 & 18.2 \\
\hline & casi nunca & 9 & 10.2 \\
\hline & a veces & 21 & 23.9 \\
\hline & Casi siempre & 17 & 19.3 \\
\hline & Siempre & 25 & 28.4 \\
\hline & Total & 88 & 100.0 \\
\hline
\end{tabular}

Tabla 5 Implementa programas de reducción del consumo del agua

Fuente: Elaboración Propia

De las personas encuestadas, 18 personas respondieron que nunca implementan programas de reducción del consumo del agua, siendo el $18.2 \%$, nueve personas respondieron que casi nunca lo hacen, correspondiendo al $10.2 \%, 21$ personas respondieron que a veces lo hacen, correspondiendo al 23.9\%, 17 personas respondieron que casi siempre, correspondiendo al $19.3 \%$ y 25 personas respondieron que siempre correspondiendo al $28.4 \%$.

\begin{tabular}{|c|l|r|r|}
\cline { 2 - 4 } \multicolumn{1}{c|}{} & Frecuencia & Porcentaje \\
\hline \multirow{4}{*}{ Válidos } & Nunca & 28 & 31.8 \\
\cline { 2 - 4 } & casi nunca & 3 & 3.4 \\
\cline { 2 - 4 } & a veces & 20 & 22.7 \\
\cline { 2 - 4 } & Casi siempre & 18 & 20.5 \\
\cline { 2 - 4 } & Siempre & 18 & 20.5 \\
\cline { 2 - 4 } & No contestó & 1 & 1.1 \\
\cline { 2 - 4 } & Total & 88 & 100.0 \\
\hline
\end{tabular}

Tabla 6 Diseña productos y empaques que pueden ser reusados, reparados o reciclado

Fuente: Elaboración Propia

Donde, 28 personas respondieron que nunca diseñan productos y empaques que pueden ser reusados, reparados o reciclados, siendo el $31.8 \%$, tres personas respondieron que casi nunca lo hacen, correspondiendo al $3.4 \%, 20$ personas respondieron que a veces lo hacen, correspondiendo al $22.7 \%, 18$ personas respondieron que casi siempre, correspondiendo al 20.5\%, 18 personas respondieron que siempre, correspondiendo al $20.5 \%$ y una persona no respondió a la pregunta siendo el $1.1 \%$.

\begin{tabular}{|c|l|r|r|}
\hline \multicolumn{1}{|c}{} & Frecuencia & Porcentaje \\
\hline \multirow{4}{*}{ Válidos } & Nunca & 38 & 43.2 \\
\cline { 2 - 4 } & casi nunca & 14 & 15.9 \\
\cline { 2 - 4 } & a veces & 13 & 14.8 \\
\cline { 2 - 4 } & Casi siempre & 12 & 13.6 \\
\cline { 2 - 4 } & Siempre & 11 & 12.5 \\
\cline { 2 - 4 } & Total & 88 & 100.0 \\
\hline
\end{tabular}

Tabla $\quad 7$ Se $\quad$ realizan periódicamente auditorías medioambientales

Fuente: Elaboración Propia
Respecto a la pregunta, si se realizan periódicamente auditorías ambientales, 38 personas respondieron que nunca las realizan, siendo el 43.2\%, 14 personas respondieron que casi nunca lo hacen, correspondiendo al $15.9 \%, 13$ personas respondieron que a veces, correspondiendo al 14.8\%, 12 personas respondieron que casi siempre, correspondiendo al $13.6 \%, 11$ personas respondieron que siempre, correspondiendo al $12.5 \%$ y una persona no respondió a la pregunta siendo el $1.1 \%$.

\begin{tabular}{|c|l|r|r|}
\hline \multicolumn{1}{|c}{} & Frecuencia & Porcentaje \\
\hline \multirow{4}{*}{ Válidos } & Nunca & 32 & 36.4 \\
\cline { 2 - 4 } & casi nunca & 14 & 15.9 \\
\cline { 2 - 4 } & a veces & 21 & 23.9 \\
\cline { 2 - 4 } & Casi siempre & 7 & 8.0 \\
\cline { 2 - 4 } & Siempre & 13 & 14.8 \\
\cline { 2 - 4 } & No contestó & 1 & 1.1 \\
\cline { 2 - 4 } & Total & 88 & 100.0 \\
\hline
\end{tabular}

Tabla 8 Adopta medidas de diseño de productos/ servicios ecológicos

Fuente: Elaboración Propia

Los encuestados se manifestaron de la siguiente forma: 32 personas respondieron que nunca adoptan medidas de diseño de productos/servicios ecológicos, siendo el $36.4 \%, 14$ personas respondieron que casi nunca lo hacen, correspondiendo el 15.9\%, 21 personas respondieron que a veces, correspondiendo al $23.9 \%$, siete personas respondieron que casi siempre, correspondiendo al $8 \%, 13$ personas respondieron que siempre correspondiendo al $14.8 \%$ y una persona no respondió a la pregunta siendo el $1.1 \%$.

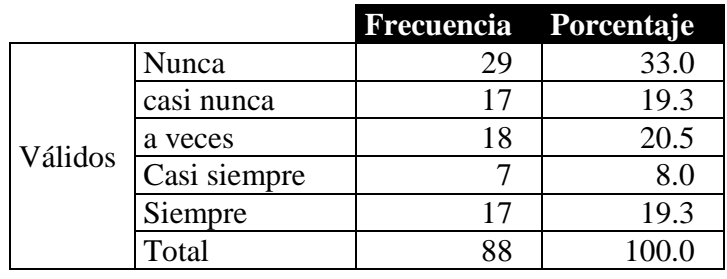

Tabla 9 Adopta programas para el uso de energías alternativas

Fuente: Elaboración Propia

De las 88 encuestas aplicadas, 29 personas respondieron que nunca adoptan programas para el uso de energías de alternativas, siendo el 33\%, 17 personas respondieron que casi nunca lo hacen, correspondiendo al $19.3 \%, 18$ personas respondieron que a veces, correspondiendo al $20.5 \%$, siete personas respondieron que casi siempre, correspondiendo al $8 \%$ y 17 personas respondieron que siempre correspondiendo al $19.3 \%$.

ACEVES-LÓPEZ, Jesús Nereida, GONZÁLEZ-NAVARRO, Nora Edith y NAVARRO-ARVIZU, Elba Myriam. Acciones ambientales: Responsabilidad Social en MiPyMes. Revista del Desarrollo Urbano y Sustentable. 2019 


\begin{tabular}{|c|c|c|c|}
\hline & & Frecuencia & Porcentaje \\
\hline \multirow{7}{*}{ Válidos } & Nunca & 29 & 33.0 \\
\hline & casi nunca & 11 & 12.5 \\
\hline & a veces & 19 & 21.6 \\
\hline & Casi siempre & 11 & 12.5 \\
\hline & Siempre & 17 & 19.3 \\
\hline & No contestó & 1 & 1.1 \\
\hline & Total & 88 & 100.0 \\
\hline
\end{tabular}

Tabla 10 Accede voluntariamente las regulaciones medioambientales legales

Fuente: Elaboración Propia

En relación a esta pregunta, 29 personas respondieron que nunca acceden voluntariamente las regulaciones medioambientales legales, siendo el 33\%, 11 personas respondieron que casi nunca lo hacen, correspondiendo al $12.5 \%, 19$ personas respondieron que a veces, correspondiendo al $21.6 \%$, once personas respondieron que casi siempre, correspondiendo al $12.5 \%$ y 17 personas respondieron que siempre, correspondiendo al $19.3 \%$ y una persona no respondió a la pregunta siendo el $1.1 \%$.

\section{Referencias}

Ancer, J. (2013). UNIVERSIDAD AUTONOMA DE NUEVO LEON SOCIALMENTE RESPONSABLE CON EL MEDIO AMBIENTE. Revista Mexicana de Ciencias Forestales. 4 (17)

Comisión de las Comunidades Europeas. (2001). Libro verde: Fomentar un marco europeo para la responsabilidad social de las empresas. Bruselas. Recuperado de: https://eurlex.europa.eu/legal-

content/ES/TXT/?qid=1488204560202\&uri=C ELEX:52001DC0366

De la Rosa, M. (2014). Restos y oportunidades del desarrollo sustentable y la responsabilidad social. Universidad de Sonora.

Gómez, J. (2014). DEL DESARROLLO SOSTENIBLE A LA SUSTENTABILIDAD AMBIENTAL. Revista Facultad de Ciencias Económicas: Investigación y Reflexión, 22(1). Recuperado el 22 de mayo, 2019, de: http://www.scielo.org.co/scielo.php?script=sci_ arttext\&pid=S0121-

$68052014000100009 \& \operatorname{lng}=\mathrm{en} \& \operatorname{tlng}=\mathrm{en}$

López de Medina, L. (2002). La empresa, el medio ambiente y la responsabilidad social. Revista Galega de Economía, 11(2).
López, O. y Gil M., (2014). Responsabilidad Social Empresarial. Gil.

Pérez, G. (2009). LA RESPONSABILIDAD CIVIL POR DAÑOS AL MEDIO AMBIENTE EN EL DERECHO COMPARADO. Prolegómenos. Derechos y Valores, XII (23), $35-45$.

Pulgarín, H. A., y Leal, L. L., (s.f.) Educación ambiental: eje estratégico para el desarrollo sustentable. Libro Ciencia de la Sustentabilidad y sus Disciplinas.

Raufflet, E. (2014). Responsabilidad corporativa y desarrollo sostenible: historia y algunas líneas para el contexto Mexicano. En Gil, M y Morales, E, (coord.), Sustentabilidad y Responsabilidad Social en las organizaciones. Editorial Hess.

Robles, M. M., Y Encinas, M. C., (2014). Dilema conceptual entre el desarrollo sustentable y la responsabilidad social. En De la Rosa, M, (coord.). Restos y oportunidades del desarrollo sustentable y la Responsabilidad Social. Universidad Autoónoma de Aguas Calientes y UNISON.

Salamanca, C. (2016). Sustentabilidad y corporaciones autónomas regionales ambientales en Boyacá- Colombia. Apuntes del Cenes. 35(61). Recuperado el 15 de julio, 2019, de:

http://www.scielo.org.co/scielo.php?script=sci_ arttext\&pid=S0120-

$30532016000100004 \& \operatorname{lng}=$ en \&tlng=en.

Wolfensberg, L. (2005). Sustentabilidad y desarrollo: suficiente siempre. Universidad Anáhuac Del Sur S.C. en coedición con Editorial Miguel Ángel Porrúa. Recuperado el 15 de Julio, 2019, de: https://ebookcentral.proquest.com/lib/biblioitso nsp/reader.action?docID=3174826 